\title{
Site-specific associations of muscle thickness with bone mineral density in middle-aged and older men and women
}

\author{
T Abe ${ }^{1,3}$, JP Loenneke ${ }^{1}$, KC Young, ${ }^{2,6}$, VK Nahar ${ }^{1,4,5}$, KM Hollaway ${ }^{2}$, CD Stover ${ }^{2}$, \\ MA Ford ${ }^{1}$, MA Bass ${ }^{1}$, SG Owens ${ }^{1}$, M Loftin ${ }^{1}$ \\ ${ }^{1}$ Department of Health, Exercise Science, and Recreation Management, School of Applied Sciences, Kevser Ermin \\ Applied Physiology Laboratory, The University of Mississippi, Oxford, MS, USA \\ ${ }^{2}$ Department of Human Performance Studies, College of Education, Wichita State University, Wichita, KS, USA \\ ${ }^{3}$ Department of Sports and Life Sciences, National Institute of Fitness and Sports in Kanoya, \\ Kanoya, Kagoshima, Japan \\ ${ }^{4}$ Department of Health, Physical Education, and Exercise Science, Lincoln Memorial University, \\ Harrogate, TN, USA \\ ${ }^{5}$ Department of Dermatology, The University of Mississippi Medical Center, Jackson, MS, USA \\ ${ }^{6}$ Department of Cell Biology and Physiology, Edward Via College of Osteopathic Medicine - Auburn Campus, \\ Auburn, AL, USA
}

Received: February 5, 2015

Accepted: May 5, 2016

It is unknown whether age-related site-specific muscle loss is associated with areal bone mineral density (aBMD) in older adults. To examine the relationships between aBMD and whole-body muscle thickness distribution, 97 healthy adults (46 women and 51 men) aged 50-78 years volunteered. Total and appendicular lean soft tissue mass, aBMD of the lumbar spine (LS-aBMD) and femoral neck (FN-aBMD) were determined using dual-energy X-ray absorptiometry. Muscle thickness (MT) was measured by ultrasound at nine sites of the body (forearm, upper arm, trunk, upper leg, and lower leg). Relationships of each co-variate with aBMD were tested partialling out the effect of age. aBMD was not correlated with either MT of the trunk or anterior lower leg in either sex. In men, significant and relatively strong correlations were observed between anterior and posterior upper arms, posterior lower leg, and anterior upper leg MT and LS-aBMD or FN-aBMD. In women, significant correlations were observed between anterior and posterior upper legs, posterior lower leg, and anterior upper arm MT and FN-aBMD. LS-aBMD was only correlated with forearm and posterior upper leg MT in women. In conclusion, the site-specific association of MT and aBMD differs between sexes and may be associated with the participants' daily physical activity profile.

Keywords: bone strength, whole-body muscle distribution, B-mode ultrasound, sarcopenia, aging

\section{Introduction}

To prevent osteoporosis, understanding the adaptation of bone to physical activity is important for developing public health strategies. Based on the current evidence, it is thought that weight-bearing exercise elicits a positive effect on bone health (9). Bone is primarily sensitive to: (1) short periods of loading with unusual strain distributions, (2) the rate and magnitude of high peak strain during loading, and (3) the variation in the way strain is distributed across a section of the bone (26). Thus, greater strain magnitude and unusual

\footnotetext{
Corresponding author: Takashi Abe, $\mathrm{PhD}$

Department of Health, Exercise Science, and Recreation Management, The University of Mississippi

215 Turner Center, University, MS 38677, USA

Phone: +1 662915 2786; Fax: +1 662915 5525; E-mail: t12abe@gmail.com
} 
strain distributions provide the most effective stimulus for bone adaptation. In line with the bone, skeletal muscle cells are stimulated under the condition of moderate- to high-load muscle contractions with high metabolic stress (20) and muscle mass is increased in response to repeated exercise (15). Furthermore, this muscle mass response is local and only occurs in the muscle group trained. Therefore, daily physical activity with optimal and unusual loads should have a positive effect on skeletal structure as well as muscle morphology.

Muscle mass loss associated with aging is greater in the lower limbs than in the upper limbs (17). Furthermore, there is a greater loss of muscle in the anterior thigh than in the posterior thigh $(1,13,21,23)$, which is defined as site-specific muscle loss $(5,8)$. This sitespecific muscle loss is observed not only in Japanese men and women (4), but also in German and American men and women $(2,7)$. Evidence exists suggesting that site-specific muscle loss may occur independently of the age-related muscle loss detected at the whole-body level (7). Site-specific thigh muscle loss may be associated with the development of physical disability in older adults (6). Similarly, age-related site-specific muscle loss may be related to bone density as described above. However, it is unknown whether there are site-specific associations of muscle thickness with areal bone mineral density (aBMD). Therefore, the purpose of this study was to examine the relationships between lumbar spine and femoral neck aBMD and muscle thickness distribution in middle-aged and older men and women.

\section{Methods}

\section{Subjects}

Approximately 97 Caucasian adults (46 women and 51 men) aged 50-78 years volunteered for this study (Table I). The participants were recruited from the university campus and surrounding area via flyers posted on campus. Prior to obtaining informed consent, a written description of the purpose of this study and its safety was distributed to potential subjects. All subjects were right-handed and free of overt chronic disease (e.g., neuromuscular, diabetes, angina, myocardial infarction, cancer, stroke, etc.) as assessed by self-report. The subjects were not taking any medications known to affect muscle, such as angiotensin II receptor blockers, steroids, or anti-diabetic drugs. Subjects being treated for mild hypertension with $\beta$-blockers or diuretics were allowed to participate in this study. Approximately $60 \%$ of the subjects (20 women and 40 men) reported participation in regular sports activity (at least twice a week) including walking, running, and cycling exercise. This study was conducted according to the Declaration of Helsinki and was approved by the University's Institutional Review Board, and written informed consent was obtained from the participants.

\section{Body composition and bone mineral density measurements}

The subjects underwent dual-energy X-ray absorptiometry (DXA) scans (Discovery A, Hologic Inc., Bedford, MA, USA) to determine the percent body fat (\%fat), total fat mass (FM, kg), total (tLM, kg) and appendicular (aLM, kg) lean soft tissue mass, and areal bone mineral density (aBMD, g/ $\mathrm{cm}^{2}$ ) of the AP lumbar spine (L1-L4) (LS-aBMD) and femoral neck of right leg's proximal femur (FN-aBMD). Because ultrasound measurements were carried out on the right side of the body, the value from the femoral neck of the right leg was used to make comparisons between aBMD and ultrasound measured muscle thickness. Quality assurance testing and calibration was performed the morning of data collection days to ensure that the DXA was operating properly. The subjects were asked to refrain from 
Table I. Body composition, MT, and aBMD in middle-aged and older women and men

\begin{tabular}{|c|c|c|c|}
\hline Variables & Women & Men & $p$-Value \\
\hline$N$ & 46 & 51 & \\
\hline Age (years) & $58(6)$ & $60(6)$ & 0.329 \\
\hline Height (m) & $1.63(0.06)$ & $1.77(0.06)$ & $<0.001$ \\
\hline Body mass (kg) & $67.7(17.3)$ & $81.3(12.6)$ & $<0.001$ \\
\hline Body mass index $\left(\mathrm{kg} / \mathrm{m}^{2}\right)$ & $25.5(5.9)$ & $25.9(3.2)$ & 0.698 \\
\hline Body fat (\%) & $31.0(7.7)$ & $19.5(4.6)$ & $<0.001$ \\
\hline Total fat mass $(\mathrm{kg})$ & $21.7(10.5)$ & $16.0(5.4)$ & $<0.001$ \\
\hline Total LM (kg) & $43.1(7.6)$ & $61.5(8.5)$ & $<0.001$ \\
\hline Appendicular LM (kg) & $18.2(3.4)$ & $27.8(3.9)$ & $<0.001$ \\
\hline Legs LM (kg) & $14.1(2.6)$ & $20.5(2.8)$ & $<0.001$ \\
\hline aBMD lumbar spine $\left(\mathrm{g} / \mathrm{cm}^{2}\right)$ & $0.95(0.12)$ & $1.04(0.14)$ & 0.001 \\
\hline aBMD femoral neck $\left(\mathrm{g} / \mathrm{cm}^{2}\right)$ & $0.72(0.10)$ & $0.79(0.12)$ & 0.003 \\
\hline \multicolumn{4}{|l|}{ Muscle thickness (cm) } \\
\hline Sum of nine sites & $29.2(2.6)$ & $35.6(2.6)$ & $<0.001$ \\
\hline Lateral forearm & $1.82(0.27)$ & $2.44(0.37)$ & $<0.001$ \\
\hline Anterior upper arm & $2.42(0.31)$ & $3.43(0.37)$ & $<0.001$ \\
\hline Posterior upper arm & $2.76(0.55)$ & $3.94(0.51)$ & $<0.001$ \\
\hline Anterior trunk & $0.95(0.16)$ & $1.32(0.29)$ & $<0.001$ \\
\hline Posterior trunk & $1.93(0.43)$ & $2.25(0.49)$ & 0.002 \\
\hline Anterior upper leg & $4.34(0.66)$ & $5.25(0.56)$ & $<0.001$ \\
\hline Posterior upper leg & $5.91(0.68)$ & $6.58(0.50)$ & $<0.001$ \\
\hline Anterior lower leg & $2.65(0.26)$ & $3.06(0.34)$ & $<0.001$ \\
\hline Posterior lower leg & $6.42(0.53)$ & $7.37(0.68)$ & $<0.001$ \\
\hline
\end{tabular}

Values are mean and standard deviation (SD). LM: lean soft tissue mass; aBMD: areal bone mineral density; and MT: muscle thickness

eating for at least $4 \mathrm{~h}$ prior to scans and were offered water ad libitum. Furthermore, the subjects were asked to refrain from moderate/vigorous exercise for at least $48 \mathrm{~h}$ prior to the scans. DXA scans were conducted immediately before or after ultrasound measurements. Test-retest reliability using intra-class correlation coefficient $\left(\mathrm{ICC}_{3,1}\right)$, standard error of measurement (SEM), and minimal difference to be considered real were previously determined from 17 subjects scanned twice $24 \mathrm{~h}$ apart from aLM $(0.99,0.21 \mathrm{~kg}$, and 
$0.58 \mathrm{~kg})$, tLM $(0.99,0.26 \mathrm{~kg}$, and $0.71 \mathrm{~kg})$ and \%fat $(0.99,0.34 \%$, and $0.95 \%)$, LS-aBMD $\left(0.99,0.011 \mathrm{~g} / \mathrm{cm}^{2}\right.$, and $\left.0.031 \mathrm{~g} / \mathrm{cm}^{2}\right)$, and FN-aBMD $\left(0.99,0.007 \mathrm{~g} / \mathrm{cm}^{2}\right.$, and $\left.0.019 \mathrm{~g} / \mathrm{cm}^{2}\right)$.

Body mass and standing height were measured to the nearest $0.1 \mathrm{~kg}$ and $0.1 \mathrm{~cm}$, respectively, using a height scale and an electronic weight scale. Body mass index (BMI) was defined as body mass $(\mathrm{kg}) /$ height $^{2}\left(\mathrm{~m}^{2}\right)$.

\section{Muscle thickness measurements}

Muscle thickness (MT) was measured using B-mode ultrasound (SSD-500, Aloka, Tokyo, Japan) at nine sites (lateral forearm [at 30\% proximal between the styloid process and the head of the radius], anterior (biceps) and posterior (triceps) upper arms [at $60 \%$ distal between the lateral epicondyle of the humerus and the acromial process of the shoulder], anterior (quadriceps) and posterior (hamstring) upper legs [midway between the lateral condyle of the femur and greater trochanter], anterior (tibialis anterior) and posterior (triceps surae) lower legs [at 30\% proximal between the lateral malleolus of the fibula and the lateral condyle of the tibia], and anterior (rectus abdominis) [about $3 \mathrm{~cm}$ lateral to the umbilicus] and posterior trunks (latissimus dorsi) [about $5 \mathrm{~cm}$ below to the inferior angle of the scapula]) on the right side of the body as previously described (3). After the measurement of limb length using anatomical landmarks described above, all the measurement sites were marked with a marker pen. The measurements were taken, while the subjects stood quietly with their elbows and knees extended and relaxed. A linear transducer with a 5-MHz scanning head was coated with water-soluble transmission gel to provide acoustic contact and reduce pressure by the scanning head to achieve a clear image. The scanning head was placed on the skin surface of the measurement site using the minimum pressure required, and cross-sections of each muscle were imaged. Images from each site were printed (SONY UP-897MD, Tokyo, Japan), and the values of each site were used for data analysis. The subcutaneous adipose tissuemuscle interface and muscle-bone interface were identified from the ultrasonic image, and the distance between the two interfaces was accepted as MT for limb muscles. For measurements in trunk, MT was defined as the distance between the adipose tissue-muscle interface and the deep muscle fascia interface. Summation of MT in measured nine sites (sum of 9 MT) was calculated as an index of total muscle mass (25). In addition, the ratio of anterior to posterior (A:P) upper leg MT was calculated as an index of site-specific thigh muscle loss (8). Test-retest reliability of MT measurements using $\mathrm{ICC}_{3,1}, \mathrm{SEM}$, and minimum difference was previously determined from 15 middle-aged subjects for anterior $(0.88,0.08 \mathrm{~cm}$, and $0.22 \mathrm{~cm})$ and posterior $(0.96,0.08 \mathrm{~cm}$, and $0.22 \mathrm{~cm})$ upper arm and anterior $(0.98,0.07 \mathrm{~cm}$, and $0.19 \mathrm{~cm})$ and posterior $(0.95,0.10 \mathrm{~cm}$, and $0.28 \mathrm{~cm})$ thigh. In addition, the estimated coefficient of variation of this method from test-retest was $0.8 \%$ (19).

\section{Statistical analysis}

All data are presented as mean and standard deviation. Before comparisons were made, dependent variables were tested for normality of distribution by the Shapiro-Wilk test. The difference between women and men was tested for significance using unpaired Student's $t$-test, and if any variables were not normally distributed, then the Mann-Whitney $U$ test was used. First, Pearson product correlations were performed to assess the relationship between aBMD and body composition (FM, tLM, and aLM) or MT. As a result, a significant negative correlation was observed between age and FN-aBMD in both sexes, although age did not significantly correlate with LS-aBMD. Therefore, relationships of each co-variate with aBMD were tested partialling out the effect of age. Significance was set at $p<0.05$. 


\section{Results}

Age and BMI were similar $(p>0.05)$ between women and men, although men were taller $(p<0.001)$ and heavier $(p<0.001)$ than women. Women had higher $(p<0.001) \mathrm{FM}$ and lower tLM and aLM than men. Women also had lower aBMD of the lumbar spine $(p=0.001)$ and femoral neck $(p=0.003)$ than men. In addition, MT was higher $(p<0.001)$ in men than in women at all measured sites and summation of all the nine sites (Table I).

There were significant positive correlations between FM and LS-aBMD in women and men, although FN-aBMD did not correlate with FM. Both LS-aBMD and FN-aBMD were positively correlated to tLM and aLM in men. In women, tLM and aLM were only correlated with FN-aBMD, but not LS-aBMD. Sum of 9 MT was significantly correlated with both LS-aBMD and FN-aBMD in men. In women, sum of 9 MT was correlated with FN-aBMD, but not LS-aBMD (Table II).

No significant correlations were observed between LS-aBMD or FN-aBMD and MT at the anterior and posterior trunks and anterior lower leg in both sexes. In men, there were significant correlations between anterior upper leg MT and aBMD at the lumbar spine and femoral neck. Posterior lower leg MT was also correlated with both LS-aBMD and FNaBMD. Surprisingly, upper arm anterior and posterior MTs were significantly correlated with aBMD at both sites (Table III). In women, significant correlations were observed between posterior upper leg MT and both LS-aBMD and FN-aBMD. There were significant correlations between FN-aBMD and MT at the anterior upper leg, posterior lower leg, and anterior upper arm. A:P upper leg MT ratio was only correlated with LS-aBMD in men. In addition, forearm MT was correlated with LS-aBMD (Table III).

\section{Discussion}

The primary findings of this study were that (1) FM was correlated with LS-aBMD in middleaged and older men and women, (2) tLM and sum of 9 MT were correlated with both LS-aBMD and FN-aBMD in men but only FN-aBMD in women, and (3) site-specific associations of MT with aBMD were observed in both sexes.

Cross-sectional studies have reported that body mass correlates with aBMD in men and women, such that individuals with greater body mass have higher aBMD at weight-bearing

Table II. Age-adjusted partial correlation between aBMD and body composition

\begin{tabular}{|l|c|c|c|c|}
\hline & \multicolumn{2}{|c|}{ Women $(n=46)$} & \multicolumn{2}{c|}{ Men (n= 51) } \\
\hline & LS-aBMD & FN-aBMD & LS-aBMD & FN-aBMD \\
\hline Total fat mass & $0.354 *$ & 0.216 & $0.317^{*}$ & 0.178 \\
\hline Total LM & 0.262 & $0.368^{* *}$ & $0.479 * * *$ & $0.338^{*}$ \\
\hline Appendicular LM & 0.217 & $0.362^{*}$ & $0.462^{* * *}$ & $0.351^{*}$ \\
\hline Sum of 9 MT & 0.179 & $0.407 * *$ & $0.479 * * *$ & $0.444^{* *}$ \\
\hline
\end{tabular}

LM: lean mass; MT: muscle thickness; LS-aBMD: areal bone mineral density at lumbar spine; and FN-aBMD: areal bone mineral density at femoral neck. ${ }^{*} p<0.05 .{ }^{* *} p<0.01 .{ }^{* * *} p<0.001$ 
Table III. Age-adjusted partial correlation between aBMD and MT distribution

\begin{tabular}{|l|c|c|c|c|}
\hline & \multicolumn{2}{|c|}{ Women $(n=46)$} & \multicolumn{2}{c|}{ Men (n= 51) } \\
\hline & LS-aBMD & FN-aBMD & LS-aBMD & FN-aBMD \\
\hline Lateral forearm MT & $0.332^{* *}$ & 0.161 & 0.251 & 0.210 \\
\hline Anterior upper arm MT & 0.180 & $0.384^{* *}$ & $0.447^{* * *}$ & $0.384^{* *}$ \\
\hline Posterior upper arm MT & 0.010 & 0.262 & $0.369^{* *}$ & $0.396^{* *}$ \\
\hline Anterior trunk MT & -0.257 & -0.097 & 0.097 & 0.166 \\
\hline Posterior trunk MT & 0.045 & 0.264 & 0.220 & 0.213 \\
\hline Anterior upper leg MT & 0.052 & $0.347^{* *}$ & $0.441^{* * *}$ & $0.349^{* *}$ \\
\hline Posterior upper leg MT & $0.308^{*}$ & $0.404^{* *}$ & 0.038 & 0.116 \\
\hline Anterior lower leg MT & 0.153 & 0.160 & 0.204 & 0.158 \\
\hline Posterior lower leg MT & 0.184 & $0.366^{* *}$ & $0.448^{* * *}$ & $0.364^{* *}$ \\
\hline A:P upper leg MT ratio & 0.203 & 0.061 & $0.377^{* *}$ & 0.257 \\
\hline
\end{tabular}

MT: muscle thickness; LS-aBMD: areal bone mineral density at lumbar spine; FN-aBMD: areal bone mineral density at femoral neck; and A:P: anterior to posterior. ${ }^{*} p<0.05$. ${ }^{* *} p<0.01 .{ }^{* * *} p<0.001$

sites $(10,11)$. Body mass consists of two major components: fat mass and fat-free mass (or tLM). A recent meta-analysis has demonstrated that both tLM and FM are significantly associated with aBMD, but tLM is more important than FM (14). In this study, the relationship between aBMD and sum of $9 \mathrm{MT}$, an index of whole-body muscle mass (25), is stronger than the relationship between aBMD and FM. Thus, our findings agree with previous research (14) that whole-body muscle mass has a greater influence on aBMD than FM, suggesting that physical activity is an important component for preventing bone loss in middle-aged and older adults.

In addition, we also found significant associations between site-specific MT and aBMD measurements in middle-aged and older men and women. Specifically, our results revealed that anterior upper arm (biceps) MT as well as anterior upper leg (quadriceps) MT is associated with both LS-aBMD and FN-aBMD in men, but only FN-aBMD in women. In a typical pattern of daily life, the anterior upper leg muscle is active for only a short period of time (1-3 h) and at relatively low intensities (3-11\% of maximum voluntary isometric contraction) (18). Previously, we have shown that the duration of vigorous daily physical activity is positively correlated with anterior upper leg MT, but not with light or moderate physical activity (22). These results support our findings as well as our previous studies $(12,28)$ that greater strain magnitude via vigorous daily activity may provide an effective osteoanabolic stimulus for men and women. On the other hand, it is unclear whether there are direct or indirect relationships between the upper extremity MT and aBMD. However, it is expected that the anterior upper arm (elbow flexor) MT is probably related to lifting and holding/carrying load during normal activities of daily living, which may differ between men and women. Thus, the tentative gained body mass from the external load may be acutely producing greater strain magnitude or unaccustomed strain 
distribution in the load-bearing lumbar spine and hip, which may stimulate bone in those regions. This type of physical work (including isometric contraction) may also stimulate working muscles in the upper extremity (27). A five-year longitudinal follow-up study reported that the rate of decline in elbow flexion strength was greater in subjects who decreased the overall physical activity compared to those who remained active or increased activity (24). In men, our findings showed that posterior upper arm MT was also associated with both LS-aBMD and FN-aBMD. On average, anterior to posterior upper arm MT ratio is relatively constant among middle-aged and older groups in men and women (4) and there is a significant correlation between anterior and posterior upper arm MTs in both sexes (men; $r=0.358$, women; $r=0.204$, both $p<0.001$ ) (4). In this study, however, anterior upper arm MT was significantly correlated to posterior upper arm MT in men, but not in women (data not shown). These results suggest that concurrent muscle adaptations in the anterior and posterior upper arm muscles may be observed in men, but not in women. These muscle adaptations may be a reflection of the correlations between upper arm MT and aBMD.

Our results indicate that posterior upper leg MT did not significantly correlate to LS-aBMD and FN-aBMD in men, although anterior upper leg MT correlated to both aBMD. As a result, the anterior to posterior upper leg MT ratio was significantly associated with LS-aBMD, but not FN-aBMD. In women, on the other hand, both anterior and posterior upper leg MTs were significantly correlated to LS-aBMD and FN-aBMD, except for the relationship between anterior upper leg MT and LS-aBMD (Table III). Therefore, there was no significant correlation between the ratio of anterior to posterior upper leg MT and aBMD in women ( $r=0.203$ and $r=0.061$, respectively). In addition, a significant correlation was observed between forearm MT and LS-aBMD in women only. These apparent sex differences are unexplained but may be due to physical activity profiles in daily life. Similarly, a previous longitudinal study investigating the relationships between the changes in BMD and physical fitness in middle-aged and older women reported that physical fitness and local muscle strength may be associated with BMD reduction at each body site (16). Therefore, the difference in physical fitness and local muscular strength may relate to the differences in BMD-MT relationships between men and women.

It is expected that muscle activation patterns in each muscle during daytime activity may support our present results. However, we did not measure exercise intensity and duration during daily life using electromyography and/or an accelerometer. In addition, the sample size of this study is relatively small, although this phenomenon may be similar when the sample size is increased. An additional study is needed to address these potential issues.

In conclusion, our results indicate that aBMD was significantly associated with MT of the upper and lower extremities but not the trunk, and site-specific associations of MT with aBMD differ between men and women, which may be related to the participants' daily physical activity profile.

\section{Acknowledgements}

The authors would like to thank the volunteers who participated in this study. This study received no specific grant from any funding agency in the public, commercial, or not-for-profit sectors.

\section{Conflict of interest}

The authors declare no conflict of interest. 


\section{REFERENCES}

1. Abe T, Bemben MG, Sakamaki M, Yasuda T, Kondo M, Fukunaga T: Age-related, site-specific muscle loss in 1507 Japanese men and women aged 20 to 95 years. J. Sports Sci. Med. 10, 145-150 (2011)

2. Abe T, Kawakami Y, Kondo M, Fukunaga T: Comparison of ultrasound-measured age-related, site-specific muscle loss between healthy Japanese and German men. Clin. Physiol. Funct. Imaging 31, 320-325 (2011)

3. Abe T, Kondo M, Kawakami Y, Fukunaga T: Prediction equations for body composition of Japanese adults by B-mode ultrasound. Am. J. Hum. Biol. 6, 161-170 (1994)

4. Abe T, Loenneke JP, Thiebaud RS, Fukunaga T: Age-related site-specific muscle wasting of upper and lower extremities and trunk in Japanese men and women. Age (Dordr) 36, 813-821 (2014)

5. Abe T, Loenneke JP, Thiebaud RS, Loftin M: Age-related muscle loss of the anterior and posterior thigh assessed by means of MRI/CT and ultrasound. J. Trainol. 3, 47-52 (2014)

6. Abe T, Loenneke JP, Thiebaud RS, Ogawa M, Mitsukawa N: Age-related site-specific loss in the thigh and zigzag walking performance in older men and women. Acta Physiol. Hung. 101, 488-495 (2014)

7. Abe T, Patterson KM, Stover CD, Geddam DA, Tribby AC, Lajza DG, Young KC: Site-specific thigh muscle loss as an independent phenomenon for age-related muscle loss in middle-aged and older men and women. Age (Dordr) 36, 1353-1358 (2014)

8. Abe T, Thiebaud RS, Loenneke JP, Loftin M, Fukunaga T: Prevalence of site-specific thigh sarcopenia in Japanese men and women. Age (Dordr) 36, 417-426 (2014)

9. Bolam KA, van Uffelen JG, Taaffe DR: The effect of physical exercise on bone density in middle-aged and old men: a systematic review. Osteoporos. Int. 24, 2749-2762 (2013)

10. Edelstein SL, Barrett-Connor E: Relation between body size and bone mineral density in elderly men and women. Am. J. Epidemiol. 138, 160-169 (1993)

11. Felson DT, Zhang Y, Hannan MT, Anderson JJ: Effects of weight and body mass index on bone mineral density in men and women: the Framingham study. J. Bone Miner. Res. 8, 567-573 (1993)

12. Frank AW, Labas MC, Johnston JD, Kontulainen SA: Site-specific variance in radius and tibia bone strength as determined by muscle size and body mass. Physiother. Can. 64, 292-301 (2012)

13. Frontera WR, Reid KF, Phillips EM, Krivickas LS, Hughes VA, Roubenoff R, Fielding RA: Muscle fiber size and function in elderly humans: a longitudinal study. J. Appl. Physiol. 105, 637-642 (2008)

14. Ho-Pham LT, Nguyen UD, Nguyen TV: Association between lean mass, fat mass, and bone mineral density: a meta-analysis. J. Clin. Endocrinol. Metab. 99, 30-38 (2014)

15. Hunter GR, McCarthy JP, Bamman MM: Effects of resistance training on older adults. Sports Med. 34, 329-348 (2004)

16. Iida T, Ikeda H, Shiokawa M, Aoi S, Ishizaki F, Harada T, Ono Y: Longitudinal study on physical fitness parameters influencing bone mineral density reduction in middle-aged and elderly women: bone mineral density in the lumbar spine, femoral neck, and femur. Hiroshima J. Med. Sci. 61, $23-28$ (2012)

17. Janssen I, Heymsfield SB, Wang ZM, Ross R: Skeletal muscle mass and distribution in 468 men and women aged 18-88 yr. J. Appl. Physiol. 89, 81-88 (2000)

18. Klein CS, Peterson LB, Ferrell S, Thomas CK: Sensitivity of 24-h EMG duration and intensity in the human vastus lateralis muscle to threshold changes. J. Appl. Physiol. 108, 655-661 (2010)

19. Kumagai K, Abe T, Brechue WF, Ryushi T, Takano S, Mizuno M: Sprint performance is related to muscle fascicle length in male 100-m sprinters. J. Appl. Physiol. 88, 811-816 (2000)

20. Kumar V, Atherton P, Smith K, Rennie MJ: Human muscle protein synthesis and breakdown during and after exercise. J. Appl. Physiol. 106, 2026-2039 (2009)

21. Maden-Wilkinson TM, Degens H, Jones DA, McPhee JS: Comparison of MRI and DXA to measure muscle size and age-related atrophy in thigh muscles. J. Musculoskelet. Neuronal Interact. 13, 282-290 (2013)

22. Ogawa M, Mitsukawa N, Loftin M, Abe T: Association of vigorous physical activity with age-related, sitespecific loss of thigh muscle in women: the HIREGASAKI study. J. Trainol. 1, 6-9 (2012)

23. Ogawa M, Yasuda T, Abe T: Component characteristics of thigh muscle volume in young and older healthy men. Clin. Physiol. Funct. Imaging 32, 89-93 (2012)

24. Rantanen T, Era P, Heikkinen E: Physical activity and the changes in maximal isometric strength in men and women from the age of 75 to 80 years. J. Am. Geriatr. Soc. 45, 1439-1445 (1997)

25. Sanada K, Kearns CF, Midorikawa T, Abe T: Prediction and validation of total and regional skeletal muscle by ultrasound in Japanese adults. Eur. J. Appl. Physiol. 96, 24-31 (2006) 
26. Turner $\mathrm{CH}$, Robling AG: Mechanisms by which exercise improves bone strength. J. Bone Miner. Metab. 23 (Suppl.), 16-22 (2005)

27. Welsh L, Rutherford OM: Effects of isometric strength training on quadriceps muscle properties in over 55 year olds. Eur. J. Appl. Physiol. 72, 219-223 (1996)

28. Young KC, Sherk VD, Bemben DA: Inter-limb musculoskeletal differences in competitive ten-pin bowlers: a preliminary analysis. J. Musculoskelet. Neuronal Interact. 11, 21-26 (2011) 